\title{
DIRECTED EVOLUTION REVEALS THE FUNCTIONAL SEQUENCE SPACE OF AN ADENYLATION DOMAIN SPECIFICITY CODE
}

Supporting Information

Kurt Throckmorton ${ }^{1,5}$, Vladimir Vinnik ${ }^{1,5}$, Ratul Chowdhury ${ }^{2}$, Taylor B. Cook ${ }^{3}$, Marc G. Chevrette $^{1,4}$, Costas Maranas ${ }^{2}$, Brian Pfleger ${ }^{3}$, Michael George Thomas ${ }^{1 \#}$

${ }^{1}$ Department of Bacteriology, University of Wisconsin-Madison, Madison, WI, USA

${ }^{2}$ Department of Chemical Engineering, The Pennsylvania State University, University Park, PA, USA

${ }^{3}$ Department of Chemical and Biological Engineering, University of Wisconsin-Madison, Madison, WI, USA

${ }^{4}$ Department of Genetics, University of Wisconsin-Madison, Madison, WI, USA ${ }^{5}$ Contributed equally to this work

\#Address correspondence to Michael G. Thomas, michael.thomas@wisc.edu 
Figure S1. Experimental workflow.

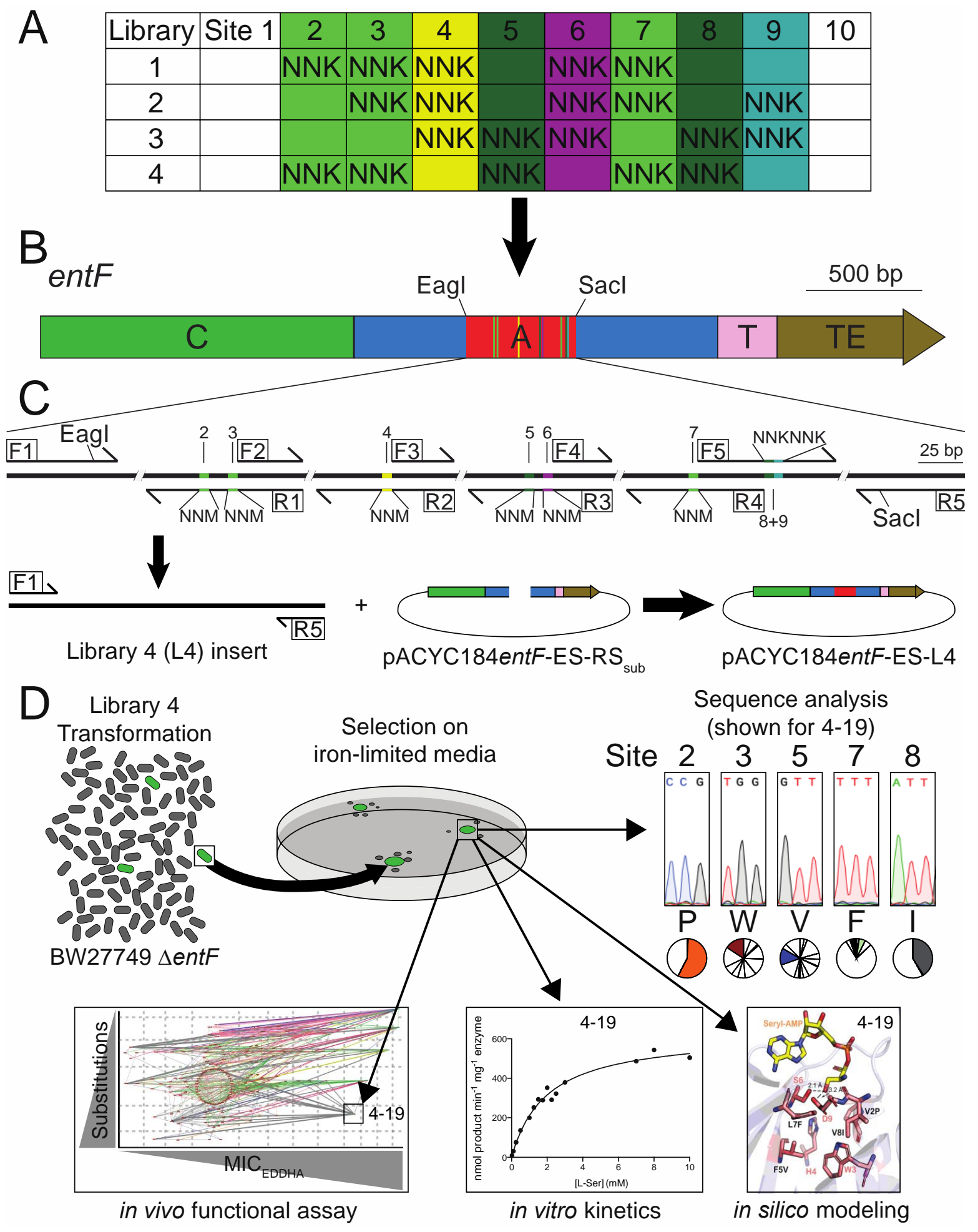


A) Table of randomized sites in each library designated by 'NNK'. Color coding is shared with Figure 1C, 1D, and 1E. Sites 4, 6, and 9 with polar residues (His, Ser, and Asp, respectively in EntF) are in yellow, purple, and teal, respectively. Sites 2, 3, and 8 with non-polar residues $\mathrm{Val}$, Trp, and Val, respectively, are in light green. Sites 5 and 8, with non-polar residues Phe and Val, respectively, are in dark green. B) Diagram of the entF gene highlighting the boundaries of the recognition subdomain (RS) of the encoded A domain (in red), flanked with unique restriction sites, Eagl and Sacl. Locations of the specificity code residues are color coded to match Figure S1A and S1C. C) Diagram of the overlap extension PCR process and cloning. The codons corresponding to the specificity code residues are represented as line segments (colored to match Figure S1A and S1B) in the template DNA (thick black line). The template DNA is amplified as five fragments by the primers shown as half arrows. The specificity code codons are replaced in the forward $(F)$ primers with NNK and MNN in the reverse $(R)$ primers. Mutagenized sites in the library insert (e.g. L4 insert) are represented as rainbow colored line segments. This process is further detailed in the experimental methods. D) An entF specificity code library (e.g. L4) is transformed into BW27749 $\Delta$ entF E. coli strain. Cells that receive a plasmid containing a mutagenized copy of entF that encodes a functional EntF variant are shown in green while cells that receive a plasmid containing a mutagenized copy of entF that encodes a non-functional EntF variant are shown in grey. Green cells can produce ENT and grow on selective plates (shown as large colonies) containing the exogenously added chelator, EDDHA, while grey cells are unable to produce ENT and are only visible as small satellite colonies nearby green cells. Once green cells have been isolated away from grey cells (refer to experimental methods), the plasmid DNA is 
harvested and sequenced to determine which residues are present at the mutagenized sites and perform sequence and statistical analyses. EntF variants (e.g. 4-19) are characterized using in vivo, in vitro, and in silico approaches. 
Figure S2. Characterization of EntF variants specificity by ATP/PPi exchange.

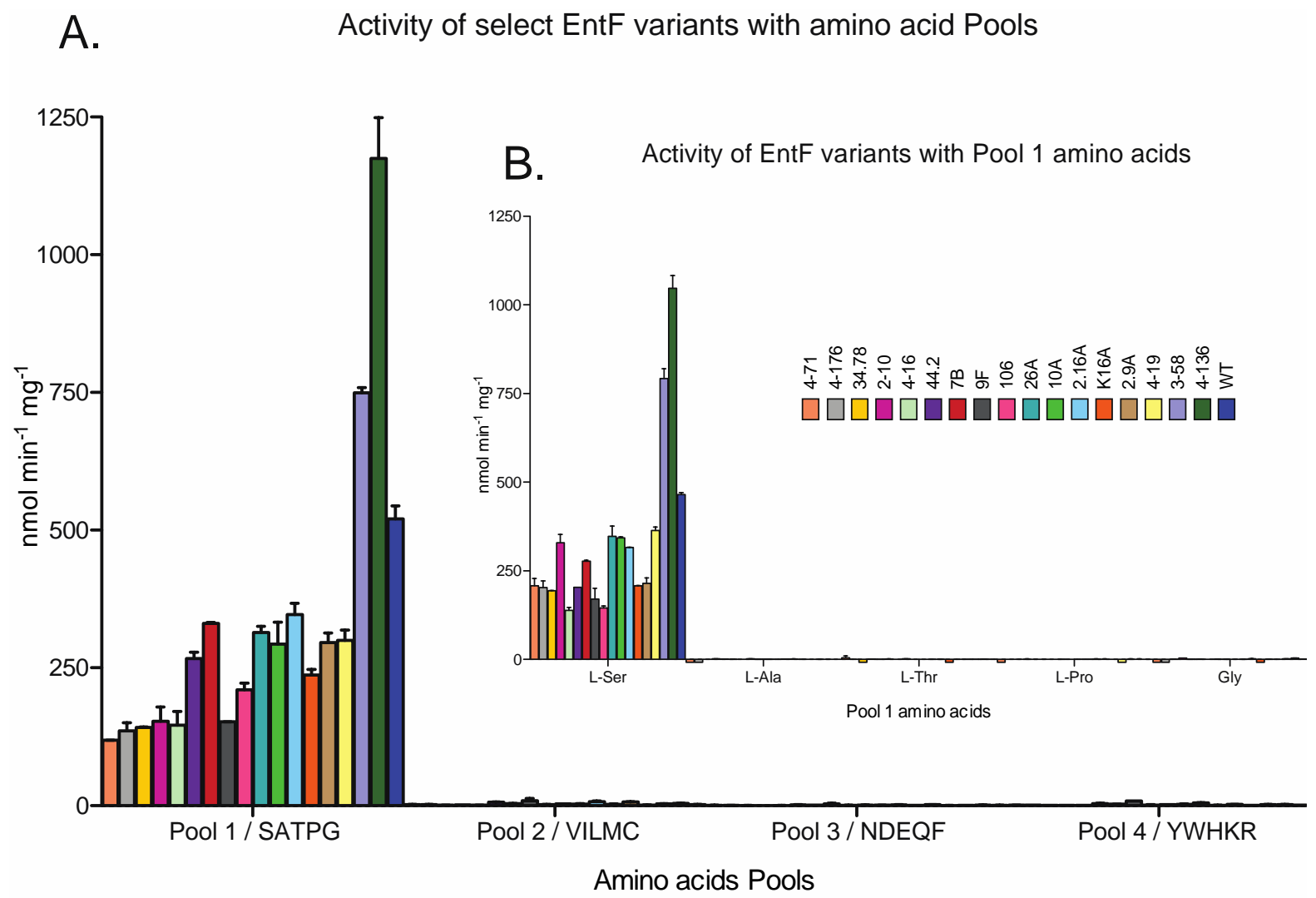

ATP/PPi exchange assays for the select EntF variants presented in Table 1 with pooled (A) or individual (B) amino acid substrates. In both panels the variants are order from left to right by increasing associated MIC EDDHA (as in Table 1) and both panels are color coded according to the key in panel B. Amino acid Pool 1: SATPG, 2: VILMC, 3: NDEQF, 4: WWHKR, 
Figure S3. Kinetics analyses of select EntF variants as determined by ATP/PPi
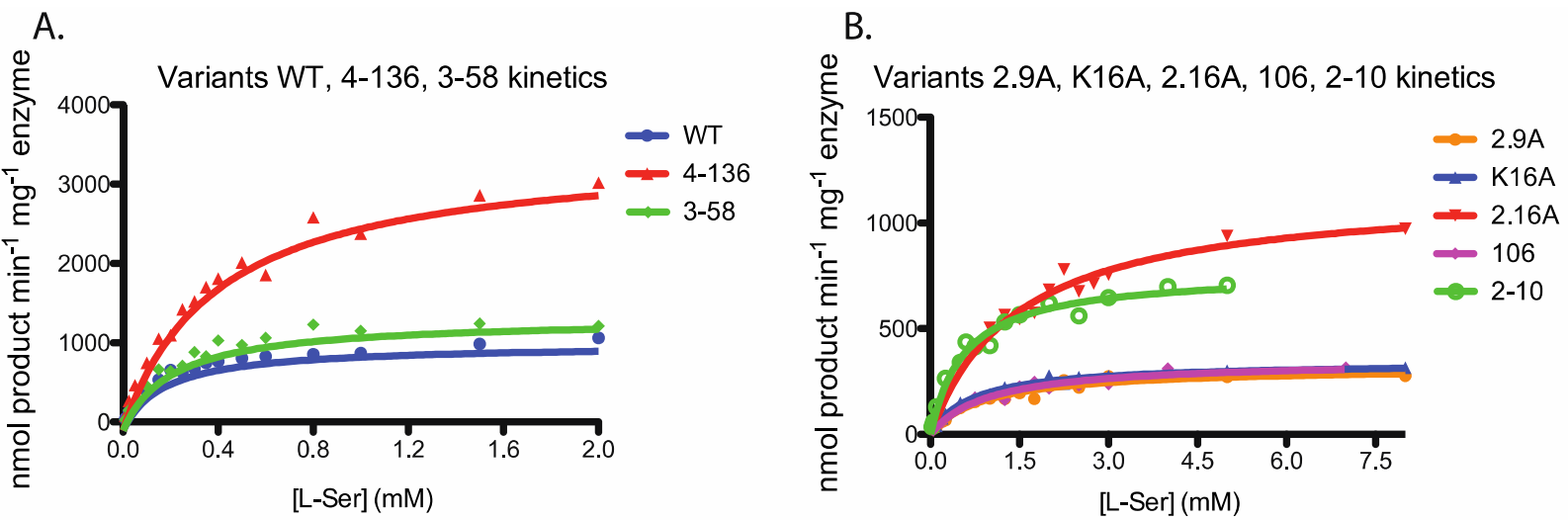

C.

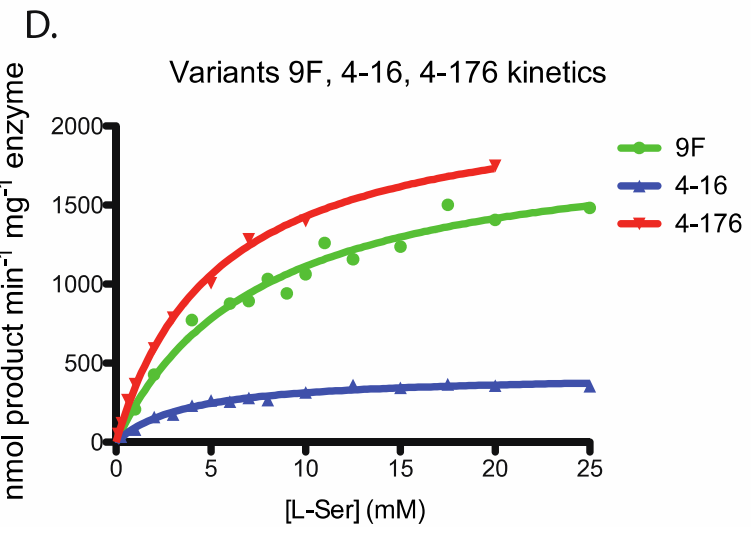

Kinetics analysis of L-Ser activation by select EntF variants presented in Table 1 as determined by ATP/PPi exchange assays. All assays were performed in the linear range for the enzyme and less than 10\% substrate-to-product conversion. Variants were grouped based on range of L-Ser concentrations used in the assay with those in panel $A$ having the lowest $K_{m}$ for $L-S e r$ and those in panel $D$ having the highest $K_{m}$ for $L-S e r$. 
Figure S4. Diversity of A domains with specificity codes matching our variants.

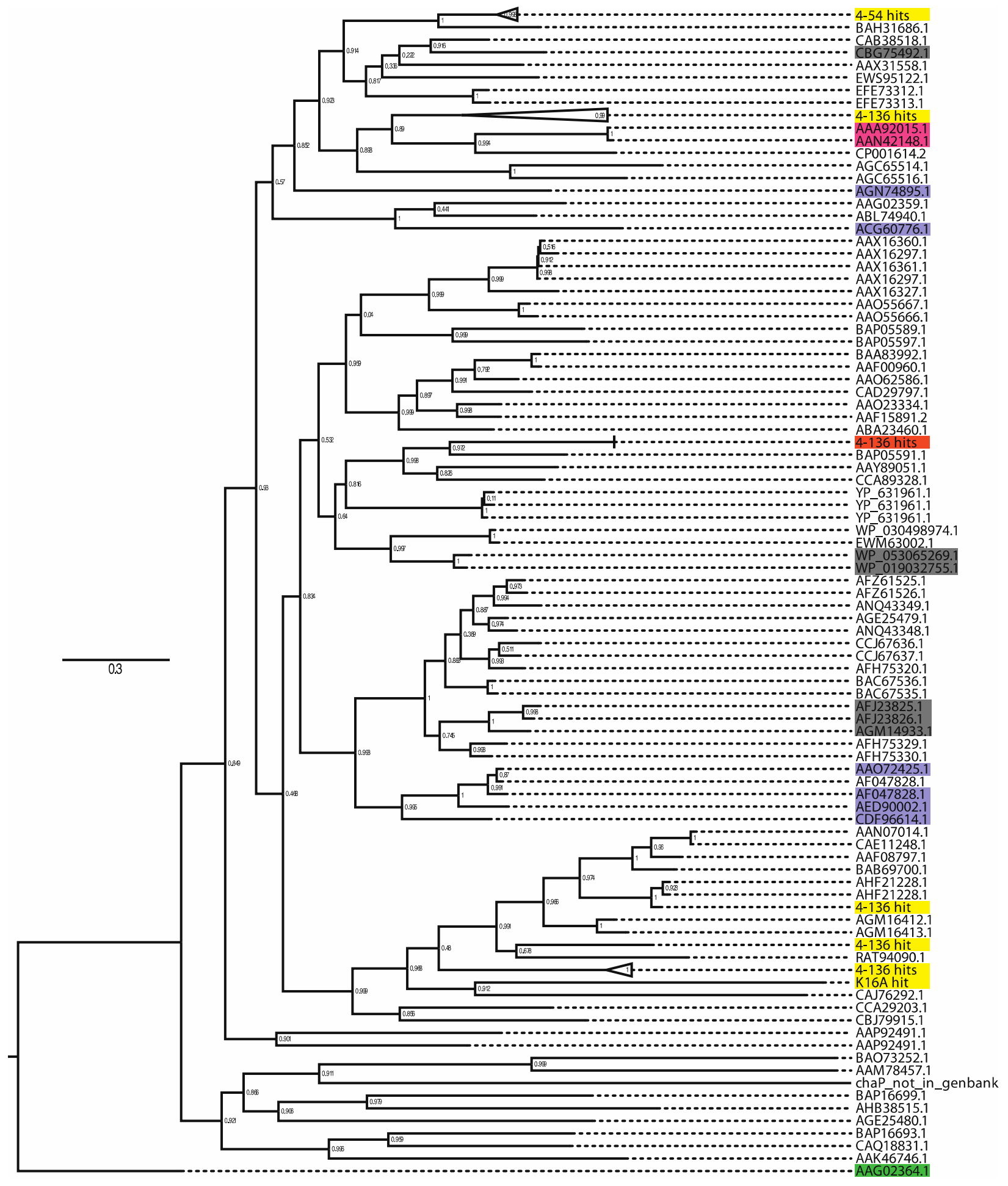


The A domains of 82 characterized, L-Ser-specific modules (Table S1), several with specificity for serine analogs or $\beta$-Ala (included as an outgroup), as well as 70 found in GenBank with predicted specificity codes matching several of our variants were aligned, and a phylogenetic tree constructed. Uncharacterized proteins with codes matching our variants K16A, 4-54, and 4-136 are highlighted in yellow, with Paenibacillus donghaensis DItA, characterized in this study, in orange. Characterized proteins with codes matching our variants $3-58$ and $4-213$ are highlighted in gray and purple, respectively. EntF is highlighted in pink, and the $\beta$-Ala-specific outgroup in green. The A domains with specificity codes matching several of our variants span this tree. 
Table S1. Characterized L-Ser-specific A domains.

\begin{tabular}{|c|c|c|c|}
\hline $\begin{array}{c}\text { GenBank } \\
\text { Accession }\end{array}$ & MIBiG ID & Metabolite & Specificity Code \\
\hline AAX16360.1 & $\mathrm{N} / \mathrm{A}$ & Pyoverdine & DVWHVSLIDK \\
\hline AAX16297.1 & $\mathrm{N} / \mathrm{A}$ & Pyoverdine & DVWHVSLIDK \\
\hline AAX16297.1 & $\mathrm{N} / \mathrm{A}$ & Pyoverdine & DVWHVSLIDK \\
\hline AAX16361.1 & $\mathrm{N} / \mathrm{A}$ & Pyoverdine & DVWHVSLIDK \\
\hline AAO72425.1 & BGC0000438 & Syringopeptin & DVWHLSLIDK \\
\hline AAX31558.1 & BGC0000336 & Daptomycin & DVWH I SLVDK \\
\hline AGC65514.1 & $\mathrm{N} / \mathrm{A}$ & Thalassospiramide $b$ & DVWHFSLVDK \\
\hline CCA29203.1 & BGC0000955 & Althiomycin & DVWHFSLIDK \\
\hline AFH75329.1 & BGC0000398 & Orfamide & DVWHMSLVDK \\
\hline AFH75330.1 & BGC0000398 & Orfamide & DVWHMSLVDK \\
\hline AGC65516.1 & BGC0001050 & Thalassospiramide a & DVWHFSLVDK \\
\hline AAO55667.1 & $\mathrm{N} / \mathrm{A}$ & Pyoverdine & DVWHVSLIDK \\
\hline AAA92015.1 & $\mathrm{N} / \mathrm{A}$ & Enterobactin & DVWHFSLVDK \\
\hline BAC67536.1 & BGC0000305 & Arthrofactin & DVWHMSLVDK \\
\hline CAB38518.1 & BGC0000315 & Calcium dependent antibiotic & DVWHFSLVDK \\
\hline AAO23334.1 & BGC0000397 & Nostocyclopeptide & DVWH I SLIDK \\
\hline BAH31686.1 & BGC0000371 & Heterobactin & DVPHNSLVDK \\
\hline CCJ67636.1 & BGC0000447 & Tolaasin & DVWHMSHVEK \\
\hline CCJ67637.1 & BGC0000447 & Tolaasin & DVWHMSLVDK \\
\hline BAP16699.1 & BGC0000376 & Jbir-34, jbir-35 & DMWNTGLVHK \\
\hline AFH75320.1 & BGC0000425 & Sessilin & DVWHMSLVDK \\
\hline CDF96614.1 & BGC0001149 & Brabantamide & DVWHLSLIDK \\
\hline AGM16412.1 & BGC0000400 & Paenibacterin & DVWHFSLVDK \\
\hline AGM16413.1 & BGC0000400 & Paenibacterin & DVWHFSLVDK \\
\hline YP 631961.1 & BGC0000393 & Myxoprincomide & DVWHVSLVDK \\
\hline YP_631961.1 & BGC0000393 & Myxoprincomide & DVWHVSLVDK \\
\hline YP 631961.1 & BGC0000393 & Myxoprincomide & DVWHVSLVDK \\
\hline EFE73312.1 & BGC0000431 & Stenothricin & DVWHVSLVDK \\
\hline EFE73313.1 & BGC0000431 & Stenothricin & DVWHVSLVDK \\
\hline CBG75492.1 & BGC0000423 & Scabichelin & DVWHLSLVDK \\
\hline EWS95122.1 & BGC0000306 & Arylomycin & DVWHMSLIDK \\
\hline AAM78457.1 & $\mathrm{N} / \mathrm{A}$ & Peptaibols & DVGYLAAVYK \\
\hline AGM14933.1 & BGC0000463 & Xantholysin & DVWHLSLVDK \\
\hline AHF21228.1 & BGC0000449 & Tridecaptin & DVWHFSLVDK \\
\hline AHF21228.1 & BGC0000449 & Tridecaptin & DVWHFSLVDK \\
\hline CBJ79915.1 & BGC0001133 & Taxlllaid & DVWHFSLIEK \\
\hline AAY89051.1 & BGC0001069 & $\begin{array}{l}\text { Chivosazole } \\
\end{array}$ & DVWHFSLVDK \\
\hline AF047828.1 & BGC0000437 & Syringomycin & DLWHLSLIDK \\
\hline AF047828.1 & BGC0000437 & Syringomycin & DVWHLSLIDK \\
\hline BAC67535.1 & BGC0000305 & Arthrofactin & DVWHMSLVDK \\
\hline
\end{tabular}




\begin{tabular}{|c|c|c|c|}
\hline AAX16327.1 & $\mathrm{N} / \mathrm{A}$ & Pyoverdine & DVWHVSLIDK \\
\hline AAF08797.1 & BGC0001103 & Mycosubtilin & DVWHFSLIDK \\
\hline BAB69700.1 & BGC0001098 & Iturin & DVWHFSLIDK \\
\hline AAF15891.2 & BGC0001028 & Nostopeptolide & DVWH I SL IDK \\
\hline CP001614.2 & BGC0000451 & Turnerbactin & DVWHFSLVDK \\
\hline BAA83992.1 & $\mathrm{N} / \mathrm{A}$ & Microcystin & DVWHFSLIDK \\
\hline AFZ61525.1 & BGC0000462 & Wlip & DVWHMSLVDK \\
\hline AFZ61526.1 & BGC0000462 & Wlip & DVWHMSLVDK \\
\hline AAN07014.1 & $\mathrm{N} / \mathrm{A}$ & Bacillomycin & DVWHFSLIDK \\
\hline CAE11248.1 & BGC0001090 & Bacillomycin & DVWHFSLIDK \\
\hline AAP92491.1 & BGC0000458 & Viomycin & DVYHFSLVDK \\
\hline AAP92491.1 & BGC0000458 & Viomycin & DVRHMSMVEK \\
\hline AAN42148.1 & $\mathrm{N} / \mathrm{A}$ & Enterobactin & DVWHFSLVDK \\
\hline AAO55666.1 & $\mathrm{N} / \mathrm{A}$ & Pyoverdine & DVWHMSLIDK \\
\hline AAG02359.1 & BGC0000963 & Bleomycin & DVWHVSLVDK \\
\hline CAD29797.1 & BGC0001015 & Microcystin & DVWH I SLIDK \\
\hline ChaP (N/A) & BGC0000321 & Chaetocin & DACALGASMK \\
\hline AHB38515.1 & BGC0000345 & $\mathrm{N} / \mathrm{A}$ & DVWS I AMVHK \\
\hline ABA23460.1 & BGC0000427 & Shinorine & DVWH I SL IDK \\
\hline AED90002.1 & BGC0000443 & Thanamycin & DVWHLSLIDK \\
\hline AGN74895.1 & BGC0000459 & Griseoviridin & DVWHLSLIDK \\
\hline AFJ23825.1 & BGC0000462 & Wlip & DVWHLSLVDK \\
\hline AFJ23826.1 & BGC0000462 & Wlip & DVWHLSLVDK \\
\hline CAQ18831.1 & BGC0000954 & Ajudazol & DLYNLALVWK \\
\hline BAP05589.1 & BGC0000967 & Calyculin & DVWHFSLIDK \\
\hline BAP05591.1 & BGC0000967 & Calyculin & DVWHFSLVDK \\
\hline BAP05597.1 & BGC0000967 & Calyculin & DVWHFSLIDK \\
\hline CAJ76292.1 & BGC0000972 & Colibactin & DVWHFSLIDK \\
\hline AA062586.1 & BGC0001016 & Microcystin & DVWH I SLIDK \\
\hline AAF00960.1 & BGC0001017 & Microcystin LR & DVWHFSLIDK \\
\hline ABL74940.1 & BGC0001048 & Tallysomycin & DVWHVSLVDK \\
\hline ACG60776.1 & BGC0001058 & Zorbamycin & DVWHLSLIDK \\
\hline CCA89328.1 & BGC0001111 & Rhizopodins & DVWHFSLVDK \\
\hline AGE25479.1 & BGC0001208 & Poaeamide & DVWHMSLVDK \\
\hline AGE25480.1 & BGC0001208 & Poaeamide & DAMF LGCTYK \\
\hline WP 030498974.1 & BGC0001327 & Rakicidin & DVWH I SLIDK \\
\hline EWM63002.1 & BGC0001328 & Rakicidin & DVWH I SLIDK \\
\hline WP_053065269.1 & BGC0001330 & $\mathrm{Be}-43547 \mathrm{a} 1$ & DVWHLSLVDK \\
\hline WP_019032755.1 & BGC0001331 & $\mathrm{Be}-43547 \mathrm{a} 1$ & DVWHLSLVDK \\
\hline ANNQ43348.1 & BGC0001347 & Poaeamide b & DVWH I SLVDK \\
\hline ANQ43349.1 & BGC0001347 & Poaeamide b & DVWHMSLVDK \\
\hline RAT94090.1 & BGC0001469 & Marthiapeptide a & DVFHFSLIHK \\
\hline $\begin{array}{c}\text { AAG02364.1 ( } \beta- \\
\text { Ala outgroup) }\end{array}$ & BGC0000963 & Bleomycin & VDWVISLADK \\
\hline
\end{tabular}




\begin{tabular}{|c|c|c|c|}
\hline $\begin{array}{c}\text { BAP16693.1 } \\
(\alpha M e-S e r)\end{array}$ & BGC0000376 & Jbir-34, Jbir-35 & DAYNVSSCWK \\
\hline $\begin{array}{c}\text { BAO73252.1 }(\alpha- \\
\text { Thr/Ser) }\end{array}$ & BGC0000456 & Verlamelin & DAQT IMA IHK \\
\hline $\begin{array}{c}\text { AAK46746.1 } \\
\text { (Ser/Thr) }\end{array}$ & N/A & Dihydroaeruginoic acid & DMLNAGLVHK \\
\hline
\end{tabular}

All A domains are specific for L-Ser unless otherwise noted. AAG02364.1 is a $\beta$-Ala specific A domain included as an outgroup in Figure S4. 
Table S2. All DNA-unique ENT producer strains.

\begin{tabular}{|c|c|c|c|c|c|}
\hline Strain & Code & MICEDDHA $(\mu \mathrm{M})$ & $\begin{array}{c}\text { Ser-AMP } \\
\text { binding scores }\end{array}$ & $\begin{array}{l}\text { Non-code } \\
\text { mutations }\end{array}$ & $\begin{array}{l}\text { Rare } \\
\text { residues }\end{array}$ \\
\hline WT & DWWHFSLVDK & 850 & -389.53 & & \\
\hline $4-136$ & DWWHYSLVDK & 850 & -355.18 & & \\
\hline $4-213$ & DVWHLSLIDK & 750 & -288.17579 & & \\
\hline $4-232$ & DPWHMSLIDK & 650 & -279.75388 & & \\
\hline $4-164$ & DWWHYSWVDK & 650 & -269.13495 & & $7 \mathrm{~V}$ \\
\hline $4-19$ & DPWHVSFIDK & 600 & -366.16 & & \\
\hline $3-58$ & DVWHLSLVDK & 600 & -266.49 & & \\
\hline 4-17 & DPWHFSLVDK & 500 & -222.63136 & & \\
\hline $4-131$ & DPWHYSLIDK & 500 & -216.40647 & & \\
\hline $4-22$ & DPWHYSLVDK & 500 & -219.702 & & \\
\hline 41.60 & DVIHFSHVDK & 500 & -221.53285 & & \\
\hline $\mathrm{K} 16 \mathrm{~A}$ & DVIHFSLVDK & 500 & -238.69 & & \\
\hline $2.9 \mathrm{~A}$ & DVWHFSMVDK & 500 & -241.88 & & \\
\hline $4-39$ & DVWHWSLVDK & 500 & -179.4233 & & \\
\hline $4-147$ & DPWHASLIDK & 450 & -188.94372 & & \\
\hline 4-102 & DPWHHSLIDK & 450 & -200.66116 & & \\
\hline $4-112$ & DVVHFSLVDK & 450 & -219.702 & & \\
\hline $4-132$ & DVVHYSLVDK & 450 & -199.56265 & & \\
\hline 4-207 & DWWHTSLIDK & 450 & -215.30796 & & \\
\hline $4-51$ & DPIHLSLVDK & 400 & -169.90288 & E668K & \\
\hline $4-126$ & DPIHLSLVDK & Not measured & Not measured & & \\
\hline $4-212$ & DPIHLSLVDK & Not measured & Not measured & & \\
\hline $10 \mathrm{~A}$ & DPVHFSLVDK & 400 & -182.681 & & \\
\hline $22 \mathrm{~A}$ & DPVHFSLVDK & Not measured & Not measured & & \\
\hline F13B & DPVHFSLVDK & Not measured & Not measured & & \\
\hline 2.9 & DPVHFSLVDK & Not measured & Not measured & & \\
\hline $4-30$ & DPVHFSLVDK & 400 & -174.29692 & & \\
\hline $4-115$ & DVIHYSLVDK & 400 & -165.87501 & & \\
\hline $4-54$ & DVLHSSLVDK & 400 & -180.52181 & & \\
\hline $3-53$ & DVLHTSFIDK & 400 & -171.00139 & & \\
\hline $4-45$ & DVLHTSFVDK & 400 & -168.07203 & & \\
\hline $4-98$ & DVLHWSLVDK & 400 & -168.07203 & & \\
\hline $4-79$ & DVQHVSLIDK & 400 & -173.19841 & Q680K & \\
\hline 49.4 & DVTHFSLVDK & 400 & -183.81734 & & \\
\hline 50.7 & DVTHFSLVDK & Not measured & Not measured & & \\
\hline $2.16 \mathrm{~A}$ & DVWHFSHVDK & 400 & -208.71 & & \\
\hline $3 \mathrm{~A}$ & DVWHFSHVDK & Not measured & Not measured & & \\
\hline $15 \mathrm{~A}$ & DVWHFSWVDK & 400 & -187.84521 & & \\
\hline $4-113$ & DWWHKSLIDK & 400 & -177.59245 & & $5 \mathrm{~K}$ \\
\hline $4-155$ & DPAHFSLIDK & 350 & -166.97352 & & \\
\hline $26 \mathrm{~A}$ & DPCHFSLVDK & 350 & -144.95 & & \\
\hline $32 \mathrm{~A}$ & DPCHFSLVDK & Not measured & Not measured & & \\
\hline $4-226$ & DPCHFSLVDK & 350 & -149.03119 & & \\
\hline
\end{tabular}




\begin{tabular}{|c|c|c|c|c|c|}
\hline $4-40$ & DPCHISLIDK & 350 & -139.51077 & & \\
\hline $4-152$ & DPIHISLVDK & 350 & -171.36756 & & \\
\hline 4-146 & DPIHYSHVDK & 350 & -138.41226 & & \\
\hline $4-72$ & DPIHYSLVDK & 350 & -166.97352 & & \\
\hline 1.1 & DPLHFSHVDK & 350 & -159.65012 & & \\
\hline $4-100$ & DPLHFSHVDK & 350 & -164.04416 & & \\
\hline $4-117$ & DPQHFSLIDK & 350 & -149.03119 & & \\
\hline $4-234$ & DPQHVSLIDK & 350 & -172.46607 & & \\
\hline $4-144$ & DPTHFSLIDK & 350 & -141.70779 & & \\
\hline $4-134$ & DVCHFSLVDK & 350 & -149.03119 & $A 702 A$ & \\
\hline $4-28$ & DVCHTSLVDK & 350 & -156.35459 & & \\
\hline $4-49$ & DVCHTSLVDK & Not measured & Not measured & & \\
\hline $4-149$ & DVCHYSLVDK & 350 & -153.42523 & & \\
\hline 4-101 & DVIHCSLVDK & 350 & -149.03119 & & $5 C$ \\
\hline 2.63 & DVLHFSLVDK & 350 & -165.875 & & \\
\hline $4-233$ & DVLHFSLVDK & 350 & Not measured & & \\
\hline $2-31$ & DVLHFSLVDK & 250 & Not measured & & \\
\hline 106 & DVPHFSLVDK & 350 & -166.52 & & \\
\hline 36.21 & DVPHFSLVDK & 350 & Not measured & & \\
\hline 4-104 & DVPHFSLVDK & 350 & Not measured & & \\
\hline $4-153$ & DVPHFSLVDK & Not measured & Not measured & & \\
\hline $21 \mathrm{~A}$ & DVPHFSLVDK & Not measured & Not measured & & \\
\hline 21.45 & DVPHFSLVDK & Not measured & Not measured & P644T & \\
\hline $4-41$ & DVQHTSLIDK & 350 & -170.26905 & & \\
\hline $4-76$ & DVQHWSLVDK & 350 & -163.67799 & & \\
\hline $4-61$ & DVTHFSLIDK & 350 & -150.1297 & & \\
\hline $4-24$ & DVTHYSLVDK & 350 & -157.4531 & & \\
\hline $4-109$ & DVWHHSLIDK & 350 & -146.83417 & & \\
\hline $4-123$ & DVWHTSLIDK & 350 & -168.07203 & & \\
\hline $4-116$ & DVVHTSLVDK & 350 & -158.55161 & & \\
\hline $4-75$ & DVVHTSLVDK & Not measured & Not measured & & \\
\hline $4-190$ & DVVHTSLVDK & Not measured & Not measured & L716F & \\
\hline $4-80$ & DVHHVSLIDK & 350 & -150.1297 & & \\
\hline 4-158 & DVWHMSWVDK & 350 & -157.4531 & & $7 \mathrm{~W}$ \\
\hline $109 \mathrm{~A}$ & DPAHFSLVDK & 250 & -125.96248 & & \\
\hline $35 \mathrm{~A}$ & DPAHFSLVDK & Not measured & Not measured & & \\
\hline 2.13 & DPAHFSLVDK & Not measured & Not measured & K717E & \\
\hline $4-70$ & DPAHMSLIDK & 250 & -122.30078 & $\mathrm{H} 691 \mathrm{H}$ & \\
\hline 4-198 & DPCHISLVDK & 250 & -93.37335 & & \\
\hline 4-172 & DPCHVSLVDK & 250 & -91.5425 & & \\
\hline $4-231$ & DPCHVSLVDK & Not measured & Not measured & & \\
\hline $4-16$ & DPHHLSCIDK & 250 & -84.22 & & $3 \mathrm{H}$ \\
\hline $4-186$ & DPIHQSLVDK & 250 & -119.00525 & & $5 Q$ \\
\hline 4-177 & DPIHVSLVDK & 250 & -113.5127 & & \\
\hline 4-135 & DPIHVSLVDK & Not measured & Not measured & & \\
\hline $4-138$ & DPIHVSLVDK & Not measured & Not measured & & \\
\hline $4-142$ & DPIHVSLVDK & Not measured & Not measured & & \\
\hline $4-145$ & DPIHVSLVDK & Not measured & Not measured & & \\
\hline
\end{tabular}




\begin{tabular}{|c|c|c|c|c|c|}
\hline $4-4$ & DPLHNSMIDK & 250 & -103.25994 & & $7 \mathrm{M}$ \\
\hline $4-181$ & DPLHTSLVDK & 250 & -98.8659 & & \\
\hline $9 \mathrm{~F}$ & DPPHFSLVDK & 250 & -114.245 & & \\
\hline $4-33$ & DPPHFSLVDK & 250 & Not measured & & \\
\hline $2.5 \mathrm{~A}$ & DPPHFSLVDK & Not measured & Not measured & & \\
\hline $37 \mathrm{~A}$ & DPPHFSLVDK & Not measured & Not measured & & \\
\hline $29 A$ & DPPHFSLVDK & Not measured & Not measured & & \\
\hline $12 \mathrm{~A}$ & DPPHFSLVDK & Not measured & Not measured & & \\
\hline $24 \mathrm{~B}$ & DPPHFSLVDK & Not measured & Not measured & & \\
\hline 28.40 & DPPHFSLVDK & Not measured & Not measured & & \\
\hline 5.20 & DPPHFSLVDK & Not measured & Not measured & & \\
\hline 8.26 & DPPHFSLVDK & Not measured & Not measured & & \\
\hline $4-65$ & DPPHFSLVDK & Not measured & Not measured & & \\
\hline $4-105$ & DPPHFSLVDK & Not measured & Not measured & & \\
\hline $4-56$ & DPPHVSLVDK & 250 & -96.66888 & & \\
\hline $4-130$ & DPQHLSLIDK & 250 & -112.78036 & & \\
\hline 4-111 & DPQHLSLIDK & Not measured & Not measured & & \\
\hline $4-5$ & DPQHLSLVDK & 250 & -109.11866 & & \\
\hline 4-148 & DPQHMSLIDK & 250 & -109.48483 & & \\
\hline 4-175 & DPQHTSLIDK & 250 & -114.97738 & & \\
\hline $4-88$ & DPQHVSLVDK & 250 & -106.1893 & & \\
\hline 4-154 & DPQHVSLVDK & Not measured & Not measured & & \\
\hline $4-43$ & DPSHFSLIDK & 250 & -92.27484 & & \\
\hline $4-150$ & DPSHFSLIDK & Not measured & Not measured & & \\
\hline 22.46 & DPSHFSLVDK & 250 & -109.11866 & & \\
\hline $4-230$ & DPSHISLIDK & 250 & -120.10376 & & \\
\hline $4-26$ & DPSHVSLIDK & 250 & -121.20227 & & \\
\hline $4-140$ & DPSHYSLIDK & 250 & -111.68185 & & \\
\hline 4.74 & DPTHFSLVDK & 250 & -116.44206 & & \\
\hline 1.2 & DPTHFSLVDK & Not measured & Not measured & & \\
\hline 17.38 & DPTHFSLVDK & Not measured & Not measured & & \\
\hline $4-50$ & DPTHFSLVDK & 250 & -109.11866 & & \\
\hline $4-90$ & DPTHFSLVDK & Not measured & Not measured & & \\
\hline 4-162 & DPTHVSLIDK & 250 & -109.48483 & & \\
\hline $4-27$ & DPVHASCVDK & 250 & -109.48483 & & $7 C$ \\
\hline $4-36$ & DPVHASLVDK & 250 & -119.73759 & & \\
\hline $7 \mathrm{~B}$ & DPVHFSHVDK & 250 & -135.49 & & \\
\hline 25B & DPVHFSHVDK & Not measured & Not measured & & \\
\hline 4-78 & DPVHISLVDK & 250 & -102.89377 & F658V & \\
\hline 4-84 & DPVHISLVDK & Not measured & Not measured & & \\
\hline 4-34 & DPVHLSLVDK & 250 & -112.41419 & & \\
\hline $4-59$ & DPVHLSLVDK & Not measured & Not measured & & \\
\hline $4-127$ & DPVHMSLVDK & 250 & -120.8361 & & \\
\hline 4-173 & DPVHNSLVDK & 250 & -110.21717 & & $5 \mathrm{~N}$ \\
\hline $4-32$ & DPVHVSLVDK & 250 & -105.09079 & $\mathrm{H691H}$ & \\
\hline $4-67$ & DPVHVSLVDK & Not measured & Not measured & & \\
\hline 4-73 & DPVHVSLVDK & Not measured & Not measured & & \\
\hline $4-31$ & DPVHVSLVDK & Not measured & Not measured & & \\
\hline $4-163$ & DPVHYSHVDK & 250 & -124.13163 & & \\
\hline
\end{tabular}




\begin{tabular}{|c|c|c|c|c|c|}
\hline $4-58$ & DPVHYSLVDK & 250 & -126.32865 & & \\
\hline $4-89$ & DPVHYSLVDK & Not measured & Not measured & & \\
\hline 4-114 & DPWHFSNIDK & 250 & -126.32865 & & $7 N$ \\
\hline $4-52$ & DPWHFSQIDK & 250 & -101.79526 & & $7 Q$ \\
\hline $4-38$ & DPWHISLVDK & 250 & -100.69675 & & \\
\hline 4-107 & DPWHLSLIDK & 250 & -109.11866 & & \\
\hline $4-64$ & DPWHLSLIDK & Not measured & Not measured & & \\
\hline $4-184$ & DPWHLSLIDK & Not measured & Not measured & & \\
\hline $4-8$ & DPWHSSLIDK & 250 & -109.48483 & & \\
\hline $4-83$ & DPWHTSLIDK & 250 & -102.89377 & & \\
\hline 4-119 & DPWHTSLIDK & Not measured & Not measured & & \\
\hline 42.63 & DVAHFSLVDK & 250 & -95.57 & & \\
\hline 4-125 & DVAHFSLVDK & 250 & Not measured & A709A & \\
\hline $2-18$ & DVAHFSLVDK & Not measured & Not measured & & \\
\hline 4-139 & DVAHTSLIDK & 250 & -106.1893 & & \\
\hline $4-203$ & DVAHTSLIDK & Not measured & Not measured & & \\
\hline $4-222$ & DVAHVSLIDK & 250 & -125.23014 & $\mathrm{H} 691 \mathrm{H}$ & \\
\hline 44.2 & DVCHFSFVDK & 250 & -111.57 & & \\
\hline 4-103 & DVCHHSLIDK & 250 & -123.03312 & & \\
\hline 4-191 & DVCHHSLIDK & Not measured & Not measured & & \\
\hline $2-8$ & DVHHFSLVDK & 250 & -92.27484 & & \\
\hline $4-97$ & DVIHASFVDK & 250 & -92.27484 & & \\
\hline $4-18$ & DVIHSSLVDK & 250 & -111.31568 & & \\
\hline $4-106$ & DVIHSSLVDK & Not measured & Not measured & & \\
\hline 4-157 & DVIHYSLIDK & 250 & -120.8361 & & \\
\hline $4-29$ & DVLHCSFVDK & 250 & -98.8659 & & $5 \mathrm{C}$ \\
\hline 5.77 & DVLHFSHVDK & 250 & -111.31568 & A667S & \\
\hline $12 \mathrm{H}$ & DVLHFSMVDK & 250 & -103.99228 & & \\
\hline 63.17 & DVLHFSMVDK & 250 & -106.1893 & & \\
\hline $4-47$ & DVLHHSLVDK & 250 & -125.96248 & & \\
\hline $4-180$ & DVLHLSYIDK & 250 & -111.68185 & & $7 Y$ \\
\hline $4-66$ & DVLHMSLIDK & 250 & -94.47186 & & \\
\hline $4-35$ & DVLHTSLIDK & 250 & -107.28781 & & \\
\hline $4-60$ & DVLHTSLIDK & Not measured & Not measured & & \\
\hline $4-62$ & DVLHTSLIDK & Not measured & Not measured & & \\
\hline 4-143 & DVLHWSLIDK & 250 & -92.27484 & & \\
\hline $4-20$ & DVLHWSNIDK & 250 & -121.20227 & & $7 N$ \\
\hline $4-55$ & DVLHWSNIDK & Not measured & Not measured & & $7 N$ \\
\hline $4-48$ & DVQHHSLIDK & 250 & -99.59824 & & \\
\hline $2-51$ & DVSHFSLVDK & 250 & -101.795 & & \\
\hline $4-137$ & DVSHFSLVDK & 250 & Not measured & & \\
\hline 33.25 & DVSHFSLVDK & Not measured & Not measured & & \\
\hline 4-121 & DVSHHSLIDK & 250 & -111.68185 & & \\
\hline $4-204$ & DVSHTSLIDK & 250 & -95.57037 & & \\
\hline 4-168 & DVSHYSLIDK & 250 & -109.48483 & & \\
\hline $4-44$ & DVTHHSCVDK & 250 & -98.8659 & & $7 C$ \\
\hline $4-92$ & DVTHSSLIDK & 250 & -101.06292 & & \\
\hline $4-120$ & DVTHYSLIDK & 250 & -106.1893 & & \\
\hline
\end{tabular}




\begin{tabular}{|c|c|c|c|c|c|}
\hline $4-129$ & DVHASLVDK & 250 & -121.93461 & & \\
\hline $4-209$ & DWVHCSLIDK & 250 & -106.1893 & & $5 \mathrm{C}$ \\
\hline $2-56$ & DVVHFSVVDK & 250 & -119.73759 & & \\
\hline $3-145$ & DVVHNSLVDK & 250 & -109.11866 & & $5 \mathrm{~N}$ \\
\hline $4-82$ & DVVHTSMVDK & 250 & -124.13163 & & $7 \mathrm{M}$ \\
\hline $4-99$ & DWWHFSTVDK & 250 & -105.82313 & & $7 T$ \\
\hline $2-10$ & DWWSFSLVDK & 250 & -95.2 & & \\
\hline $4-87$ & DPIHTSLVDK & 200 & -74.69868 & & \\
\hline $4-128$ & DPIHTSLVDK & Not measured & Not measured & & \\
\hline $4-141$ & DPIHYSHIDK & 200 & -88.61314 & & \\
\hline 4-174 & DPLHCSFVDK & 200 & -84.2191 & & $5 \mathrm{C}$ \\
\hline 4-195 & DPLHISLVDK & 200 & -85.31761 & & \\
\hline $4-242$ & DPLHLSLVDK & 200 & -86.41612 & & \\
\hline $4-218$ & DPLHNSFVDK & 200 & -90.44399 & & $5 \mathrm{~N}$ \\
\hline $4-187$ & DPLHNSLIDK & 200 & -81.28974 & & $5 \mathrm{~N}$ \\
\hline $4-220$ & DPVHQSLVDK & 200 & -91.5425 & & $5 Q$ \\
\hline $4-236 a$ & DPVHTSLVDK & 200 & -98.8659 & & \\
\hline $4-69$ & DPWHWSLVDK & 200 & -99.96441 & & \\
\hline 4-192 & DPWHYSHIDK & 200 & -87.51463 & & \\
\hline $4-249$ & DVAHSSLIDK & 200 & -88.61314 & & \\
\hline $4-246$ & DVLHVSFVDK & 200 & -91.5425 & & \\
\hline $4-236 b$ & DVLHWSCIDK & 200 & -85.31761 & G686D & $7 \mathrm{C}$ \\
\hline $4-217$ & DVPHISFVDK & 200 & -86.41612 & & \\
\hline $4-185$ & DVTHLSLVDK & 200 & -102.16143 & & \\
\hline 4-122 & DVVHASHVDK & 200 & -76.8957 & & \\
\hline $4-118$ & DWWHYSTIDK & 200 & -80.92357 & & $7 T$ \\
\hline $4-176$ & DAWHMSLIDK & 150 & -54.92 & & $2 A$ \\
\hline $4-182$ & DPPHMSLVDK & 150 & -68.47379 & & \\
\hline $4-95$ & DPQHLSFIDK & 150 & -61.15039 & & \\
\hline $4-229$ & DVIHVSCIDK & 150 & -65.17826 & & $7 C$ \\
\hline $4-179$ & DVLHVSMVDK & 150 & -68.47379 & & $7 \mathrm{M}$ \\
\hline 34.78 & DVTHFSMVDK & 150 & -73.36 & & \\
\hline 43.80 & DVAHFSTVDK & 100 & -42.10955 & & \\
\hline $4-253$ & DVAHISLVDK & 100 & -51.62997 & & \\
\hline $4-248$ & DVGHTSLVDK & 100 & -56.02401 & & $3 G$ \\
\hline $4-240$ & DVIHTSAVDK & 100 & -54.9255 & & $7 \mathrm{~A}$ \\
\hline $4-71$ & DVWHISLLDK & 100 & -36.62 & & $8 \mathrm{~L}$ \\
\hline $4-223$ & DCIHYSLVDK & Not measured & Not measured & & $2 \mathrm{C}$ \\
\hline
\end{tabular}

Strains with entF copies encoding EntF variants with identical specificity codes do not have an associated MIC EDDHA measurement except for 4-233, 2-31, 36.21, 4-104, 4-33, 4-125, and 4-137, which were used to test the effect of different antibiotics on growth (experimental methods). CHARMM-based interaction energy scores (IPRO energy 
scores) were not measured for any of the protein identical EntF variants. Strain 4-223 was lost, so an MICEDDHA measurement and IPRO energy score were not obtained. No significant difference was observed in the associated MICEDDHA among specificity codeidentical EntF variants containing different antibiotic resistance markers. Rare residues are observed fewer than five times at a particular site. 
Table S3. Kinetic parameters of in vitro-characterized EntF variants.

\begin{tabular}{|c|c|c|c|}
\hline $\begin{array}{c}\text { EntF } \\
\text { variant }\end{array}$ & $\begin{array}{c}K_{\mathrm{m} \text { (L-Ser) }} \\
(\mathrm{mM})\end{array}$ & $\begin{array}{c}V_{\max } \text { (nmol } \\
\text { product/min/mg } \\
\text { protein) }\end{array}$ & $V_{\max } / K_{\mathrm{m}}$ \\
\hline WT & $0.17 \pm 0.01$ & $1087 \pm 26.61$ & 6312 \\
\hline $4-136$ & $0.4 \pm 0.04$ & $3552 \pm 138.20$ & 8958 \\
\hline $3-58$ & $0.21 \pm 0.02$ & $1412 \pm 47.29$ & 6828 \\
\hline $4-19$ & $1.98 \pm 0.22$ & $629 \pm 26.57$ & 317 \\
\hline $2.9 A$ & $0.90 \pm 0.15$ & $318 \pm 17.13$ & 355 \\
\hline K16A & $0.71 \pm 0.07$ & $340 \pm 9.38$ & 480 \\
\hline $2.16 \mathrm{~A}$ & $1.47 \pm 0.17$ & $1156 \pm 50.63$ & 789 \\
\hline $10 \mathrm{~A}$ & $2.31 \pm 0.33$ & $2148 \pm 127.80$ & 931 \\
\hline $26 \mathrm{~A}$ & $3.62 \pm 0.50$ & $2433 \pm 155.10$ & 672 \\
\hline 106 & $0.97 \pm 0.17$ & $350 \pm 20.57$ & 359 \\
\hline $9 \mathrm{~F}$ & $7.48 \pm 1.14$ & $1944 \pm 117.20$ & 260 \\
\hline $7 \mathrm{~B}$ & $3.44 \pm 0.68$ & $2221 \pm 191.40$ & 645 \\
\hline 44.2 & $2.93 \pm 0.33$ & $1167 \pm 52.95$ & 399 \\
\hline $4-16$ & $3.74 \pm 0.41$ & $429 \pm 14.41$ & 115 \\
\hline $2-10$ & $0.58 \pm 0.07$ & $767 \pm 27.53$ & 1330 \\
\hline 34.78 & $1.80 \pm 0.21$ & $1351 \pm 56.93$ & 749 \\
\hline $4-176$ & $5.38 \pm 0.35$ & $2197 \pm 59.46$ & 408 \\
\hline $4-71$ & $3.65 \pm 0.35$ & $1438 \pm 69.22$ & 394 \\
\hline
\end{tabular}


Table S4. Mutagenic primers used in library construction deviate from NNK distribution at all sites except 7 and 9.

\begin{tabular}{|c|c|c|c|c|c|c|c|c|c|c|c|c|c|c|c|}
\hline \multicolumn{4}{|c|}{ Site 2} & \multicolumn{4}{|c|}{ Site 3} & \multicolumn{4}{|c|}{ Site 4} & \multicolumn{4}{|c|}{ Site 5} \\
\hline AA & Observed & $\begin{array}{c}\text { Fold } \\
\text { change } \\
\text { over } \\
\text { NNK }\end{array}$ & $\begin{array}{l}\text { Chi- } \\
\text { square }\end{array}$ & AA & Observed & $\begin{array}{c}\text { Fold } \\
\text { change } \\
\text { over } \\
\text { NNK }\end{array}$ & $\begin{array}{l}\text { Chi- } \\
\text { square }\end{array}$ & AA & Observed & $\begin{array}{c}\text { Fold } \\
\text { change } \\
\text { over } \\
\text { NNK }\end{array}$ & $\begin{array}{l}\text { Chi- } \\
\text { square }\end{array}$ & AA & Observed & $\begin{array}{l}\text { Fold } \\
\text { change } \\
\text { over } \\
\text { NNK }\end{array}$ & $\begin{array}{l}\text { Chi- } \\
\text { square }\end{array}$ \\
\hline $\mathrm{T}$ & 22 & 1.96 & 9.92 & $P$ & 48 & 2.04 & 55.67 & $P$ & 26 & 2.21 & 17.53 & $P$ & 17 & 2.07 & 24.05 \\
\hline $\mathrm{N}$ & 12 & 1.32 & 7.01 & $\bar{T}$ & 29 & 1.86 & 8.23 & $\mathrm{~S}$ & 25 & 1.13 & 3.18 & $T$ & 13 & 2.31 & 10.23 \\
\hline $\mathrm{G}$ & 4 & 0.48 & 4.78 & $E$ & 2 & 0.41 & 5.03 & C & 2 & 0.70 & 2.53 & $\bar{Y}$ & 6 & 1.54 & 3.84 \\
\hline $\mathrm{P}$ & 18 & 1.92 & 3.86 & Q & 2 & 0.81 & 5.03 & $E$ & 2 & 0.49 & 2.53 & $\mathrm{G}$ & 1 & 0.17 & 3.68 \\
\hline V & 6 & 0.45 & 2.54 & $\mathrm{G}$ & 8 & 0.55 & 4.86 & V & 7 & 0.60 & 1.88 & $L$ & 3 & 0.69 & 3.34 \\
\hline W & 2 & 0.59 & 2.39 & $\mathrm{H}$ & 13 & 1.03 & 2.30 & $\mathrm{~L}$ & 23 & 1.18 & 1.71 & C & 0 & 0.42 & 2.75 \\
\hline $\mathrm{L}$ & 11 & 0.68 & 2.15 & V & 11 & 0.57 & 2.19 & $\mathrm{H}$ & 9 & 1.49 & 1.70 & $\mathrm{R}$ & 4 & 0.34 & 2.19 \\
\hline$S$ & 22 & 1.33 & 1.43 & $L$ & 19 & 0.96 & 1.74 & $\bar{N}$ & 9 & 0.98 & 1.70 & $\bar{M}$ & 5 & 0.65 & 1.84 \\
\hline D & 3 & 0.78 & 1.27 & K & 5 & 0.74 & 1.48 & Stop & 3 & 0.67 & 1.38 & $A$ & 3 & 0.99 & 1.14 \\
\hline$F$ & 3 & 0.65 & 1.27 & $\mathrm{~N}$ & 5 & 0.93 & 1.48 & $D$ & 3 & 0.64 & 1.38 & V & 3 & 0.37 & 1.14 \\
\hline $\bar{A}$ & 14 & 1.16 & 0.61 & $\mathrm{M}$ & 12 & 0.89 & 1.38 & $F$ & 3 & 0.94 & 1.38 & Q & $T$ & 1.01 & 1.11 \\
\hline $\mathrm{R}$ & 20 & 0.78 & 0.51 & $\mathrm{R}$ & 20 & 1.00 & 1.26 & $T$ & 3 & 1.05 & 1.38 & Stop & 4 & 0.93 & 0.57 \\
\hline $\mathrm{C}$ & 4 & 0.69 & 0.50 & $\mathrm{~S}$ & 31 & 1.31 & 1.10 & $\mathrm{~K}$ & 3 & 0.75 & 1.38 & $\mathrm{H}$ & 4 & 1.68 & 0.57 \\
\hline$T$ & 4 & 0.81 & 0.50 & Stop & 6 & 0.56 & 0.77 & W & 3 & 0.54 & 1.38 & $\mathrm{~N}$ & 4 & 1.88 & 0.57 \\
\hline $\mathrm{K}$ & 4 & 1.13 & 0.50 & W & 7 & 0.65 & 0.29 & $\mathrm{M}$ & 8 & 0.80 & 0.80 & $E$ & 2 & 0.48 & 0.20 \\
\hline Q & 4 & 1.11 & 0.50 & $\mathrm{Y}$ & 7 & 0.71 & 0.29 & $\mathrm{R}$ & 14 & 0.91 & 0.71 & $F$ & 2 & 0.89 & 0.20 \\
\hline Stop & 7 & 0.90 & 0.30 & $A$ & 15 & 1.05 & 0.26 & A & 9 & 0.95 & 0.62 & 1 & 2 & 1.09 & 0.20 \\
\hline $\mathrm{H}$ & 7 & 1.30 & 0.30 & C & 8 & 0.83 & 0.04 & G & 9 & 0.45 & 0.62 & $\mathrm{~K}$ & 2 & 1.13 & 0.20 \\
\hline$E$ & 5 & 0.67 & 0.08 & $F$ & 8 & 0.86 & 0.04 & $T$ & 14 & 1.45 & 0.46 & $\bar{W}$ & 2 & 0.25 & 0.20 \\
\hline$M$ & 5 & 0.70 & 0.08 & $\mathrm{D}$ & 9 & 0.52 & 0.02 & $\mathrm{Q}$ & 6 & 1.14 & 0.00 & $\mathrm{~S}$ & 7 & 1.44 & 0.19 \\
\hline $\bar{Y}$ & 5 & 1.05 & 0.08 & $T$ & 9 & 1.12 & 0.02 & $\bar{Y}$ & 6 & 0.87 & 0.00 & $\bar{D}$ & 3 & 0.81 & 0.02 \\
\hline TRUE & 182 & & 40.59 & TRUE & 274 & & 93.47 & TRUE & 187 & & 44.27 & TRUE & 88 & & 58.24 \\
\hline
\end{tabular}




\begin{tabular}{|c|c|c|c|c|c|c|c|c|c|c|c|c|c|c|c|}
\hline \multicolumn{4}{|c|}{ Site 6} & \multicolumn{4}{|c|}{ Site 7} & \multicolumn{4}{|c|}{ Site 8} & \multicolumn{4}{|c|}{ Site 9} \\
\hline $\mathrm{AA}$ & Observed & $\begin{array}{c}\text { Fold } \\
\text { change } \\
\text { over } \\
\text { NNK }\end{array}$ & $\begin{array}{l}\text { Chi- } \\
\text { square }\end{array}$ & AA & Observed & $\begin{array}{c}\text { Fold } \\
\text { change } \\
\text { over } \\
\text { NNK }\end{array}$ & $\begin{array}{l}\text { Chi- } \\
\text { square }\end{array}$ & $A A$ & Observed & $\begin{array}{c}\text { Fold } \\
\text { change } \\
\text { over } \\
\text { NNK }\end{array}$ & $\begin{array}{l}\text { Chi- } \\
\text { square }\end{array}$ & $\mathrm{AA}$ & Observed & $\begin{array}{c}\text { Fold } \\
\text { change } \\
\text { over } \\
\text { NNK }\end{array}$ & $\begin{array}{l}\text { Chi- } \\
\text { square }\end{array}$ \\
\hline$P$ & 29 & 2.04 & 26.97 & $\mathrm{G}$ & 9 & 0.67 & 3.72 & $\mathrm{~V}$ & 14 & 1.76 & 13.48 & $T$ & 1 & 0.53 & 3.86 \\
\hline V & 4 & 0.66 & 4.84 & $P$ & 24 & 1.49 & 2.94 & $\mathrm{P}$ & 0 & 0.25 & 5.44 & V & 10 & 1.30 & 3.27 \\
\hline W & 1 & 0.54 & 3.89 & $T$ & 24 & 1.26 & 2.94 & $\mathrm{C}$ & 6 & 1.55 & 3.96 & $\mathrm{D}$ & 5 & 1.11 & 1.63 \\
\hline $\mathrm{G}$ & 5 & 0.49 & 3.62 & $\mathrm{~L}$ & 34 & 1.12 & 2.91 & $\bar{H}$ & 0 & 0.29 & 2.72 & $\mathrm{~S}$ & 12 & 0.79 & 1.41 \\
\hline Stop & 3 & 0.72 & 1.29 & $\bar{K}$ & 4 & 0.93 & 2.36 & $\bar{A}$ & 9 & 1.01 & 2.33 & $\bar{A}$ & 3 & 0.83 & 1.27 \\
\hline$N$ & 3 & 1.04 & 1.29 & Stop & 12 & 0.99 & 1.47 & $\mathrm{~W}$ & 5 & 1.52 & 1.91 & Stop & 1 & 1.05 & 1.20 \\
\hline $\mathrm{D}$ & 8 & 0.72 & 0.91 & $\mathrm{D}$ & 12 & 0.82 & 1.47 & Stop & 1 & 0.89 & 1.09 & $M$ & 1 & 0.85 & 1.20 \\
\hline $\bar{M}$ & 8 & 0.89 & 0.91 & C & 5 & 0.69 & 1.42 & $E$ & 1 & 1.14 & 1.09 & $\mathrm{~F}$ & 4 & 1.12 & 0.47 \\
\hline $\mathrm{Y}$ & 8 & 0.85 & 0.91 & $F$ & 5 & 0.97 & 1.42 & $T$ & 1 & 1.22 & 1.09 & $\mathrm{H}$ & 4 & 0.95 & 0.47 \\
\hline$T$ & 14 & 1.50 & 0.57 & $\mathrm{H}$ & 5 & 0.99 & 1.42 & $\mathrm{~N}$ & 4 & 0.80 & 0.60 & W & 4 & 1.34 & 0.47 \\
\hline$E$ & 4 & 0.61 & 0.52 & $T$ & 5 & 0.91 & 1.42 & $\mathrm{Y}$ & 4 & 0.91 & 0.60 & $G$ & 7 & 1.46 & 0.30 \\
\hline $\mathrm{H}$ & 4 & 1.41 & 0.52 & $\bar{A}$ & 21 & 1.24 & 0.97 & $L$ & 6 & 0.75 & 0.57 & $L$ & 7 & 1.14 & 0.27 \\
\hline$T$ & 7 & 1.04 & 0.29 & $\mathrm{R}$ & 21 & 0.78 & 0.76 & $S$ & 6 & 0.98 & 0.57 & $\mathrm{R}$ & 10 & 1.15 & 0.25 \\
\hline$R$ & 15 & 0.87 & 0.27 & $E$ & 6 & 0.91 & 0.72 & $\mathrm{D}$ & 2 & 1.16 & 0.19 & C & 2 & 1.26 & 0.25 \\
\hline $\mathrm{C}$ & 5 & 0.63 & 0.09 & $\mathrm{M}$ & 10 & 1.02 & 0.28 & $\mathrm{~K}$ & 2 & 0.78 & 0.19 & $E$ & 2 & 1.19 & 0.25 \\
\hline $\mathrm{F}$ & 5 & 0.85 & 0.09 & $\mathrm{~N}$ & 10 & 0.83 & 0.28 & $\mathrm{M}$ & 2 & 1.20 & 0.19 & $\bar{N}$ & 2 & 0.70 & 0.25 \\
\hline $\mathrm{L}$ & 18 & 1.11 & 0.04 & W & 10 & 0.77 & 0.28 & $\bar{R}$ & 7 & 0.77 & 0.16 & $\bar{Q}$ & 2 & 1.01 & 0.25 \\
\hline$S$ & 18 & 1.07 & 0.04 & $\bar{Q}$ & 7 & 1.10 & 0.25 & $\mathrm{G}$ & 6 & 1.96 & 0.06 & $\mathrm{P}$ & 5 & 0.71 & 0.08 \\
\hline $\bar{A}$ & 12 & 1.03 & 0.03 & $\bar{Y}$ & 7 & 0.88 & 0.25 & $T$ & 5 & 0.70 & 0.04 & $T$ & 3 & 0.79 & 0.01 \\
\hline $\mathrm{K}$ & 6 & 0.89 & 0.01 & $\mathrm{~V}$ & 15 & 0.95 & 0.22 & $F$ & 3 & 1.39 & 0.03 & $\mathrm{~K}$ & 3 & 0.75 & 0.01 \\
\hline $\mathrm{Q}$ & 6 & 1.20 & 0.01 & $\mathrm{~S}$ & 25 & 1.10 & 0.01 & $\mathrm{Q}$ & 3 & 0.28 & 0.03 & $Y$ & 3 & 0.99 & 0.01 \\
\hline TRUE & 183 & & 47.12 & FALSE & 271 & & 27.53 & TRUE & 87 & & 36.34 & FALSE & 91 & & 17.19 \\
\hline
\end{tabular}


Table showing the nature and severity of amino acid bias in our libraries, whether from the synthesis of the NNK primers or the oePCR. One hundred unselected transformants were sequenced for each library and nucleotide usage was calculated for each base within each codon for each of the five mutagenized sites for each library. These data for individual base pairs were compared by a chi-squared test to the same data aggregated across either libraries, sites, or both, and significant deviations were found whenever the data were combined across sites, but not libraries, indicating a strong site-effect on the bias, but consistency across libraries. When comparing translated codon counts pooled across libraries but not sites, all sites except 7 and 9 deviated from the expectation of perfect NNK-randomization at a $p=0.05$ threshold, designated by 'TRUE' in the red cells. This is shown in terms of the fold-change over NNK and the contribution to the total chisquared statistic in $\mathrm{A}$, with a blue (underrepresentation) to yellow (overrepresentation) color spectrum. The bias observed for sites $2-7$ was largely complementary to that observed for sites 8 and 9, with enrichment of C's primarily at the expense of G's in the first two base pairs of codons in sites $2-7$ and the opposite for sites 8 and 9 . This is likely attributable to the use of machine-mixed primers for sites $2-7$, and hand-mixed primers for sites 8 and 9 . These biases result in the amino acid composition of our libraries being quite different for sites $2-7$ versus sites 8 and 9. 
Table S5. Covariance between multiple overlapping pairs of code sites

\begin{tabular}{|c|c|c|c|c|c|c|}
\hline A) Site 2\3 & A & L & Q & W & & \\
\hline P & 2 & 4 & 10 & 16 & & \\
\hline V & 6 & 12 & 3 & 4 & & \\
\hline B) Site 8\3 & I & P & Q & S & V & W \\
\hline I & 2 & 0 & 9 & 8 & 3 & 14 \\
\hline V & 16 & 8 & 4 & 1 & 20 & 6 \\
\hline C) Site 2\5 & H & L & S & T & V & \\
\hline P & 1 & 12 & 1 & 7 & 18 & \\
\hline V & 6 & 2 & 5 & 15 & 3 & \\
\hline
\end{tabular}

The distribution of residues in pairwise comparisons between sites 2 and $3(p<0.025)(A)$, sites 3 and $8(p<<0.01)(B)$, and sites 2 and $5(p<0.01)(C)$ in our DNA-unique L4 dataset, with codes containing residues observed fewer than five times removed. Using a chisquared test, these distributions were found to be significantly skewed from an even distribution of the possibilities at each site amongst those at the other site. Shown are the pairwise distributions with a contribution to the total chi-squared statistic of greater than $5 \%$. The number of variants containing a particular residue pair is indicated in the corresponding cell within the tables. The magnitude of the chi-squared values for each cell is indicated by shades of red; larger chi-squared values, corresponding to a more significant skew, being darker red. The yellow-shaded cell in Table A, corresponding to Pro2, Gln3, is shown despite not reaching the $5 \%$ only on account of its partner, Val2, Gln3, which did cross this threshold. The color coding between Table A and B is continuous while Table $\mathrm{C}$ is separate due to having a different degree of freedom. 
Table S6. Strains, primers, and plasmids used in this study.

\begin{tabular}{|c|c|c|}
\hline REAGENT or RESOURCE & SOURCE & IDENTIFIER \\
\hline \multicolumn{3}{|l|}{ Bacterial strains } \\
\hline $\begin{array}{l}\text { E. coli strain: } 10-\beta \\
\Delta \text { (ara-leu) } 7697 \text { araD139 fhuA } \Delta \text { lacX74 galK16 } \\
\text { galE15 e14- } \Phi 80 \mathrm{dlacZ} \text { M } 15 \text { recA1 relA1 endA1 } \\
\text { nupG rpsL (StrR) rph spoT1 } \Delta \text { (mrr-hsdRMS- } \\
\text { mcrBC) }\end{array}$ & $\begin{array}{l}\text { New England } \\
\text { Biolabs }\end{array}$ & Cat \#C3020K \\
\hline $\begin{array}{l}\text { E. colistrain: BW27749 } \\
\text { F- }, \Delta(\text { araD-araB }) 567, \Delta \text { lacZ4787(::rrnB-3), } \lambda- \\
\Delta(\text { araH-araF }) 570(:: F R T), \Delta \text { araEp531::kan, } \\
\varphi P \text { cp8araE535, rph-1, } \Delta(\text { rhaD-rhaB }) 568, \\
h s d R 514\end{array}$ & 7 & $\mathrm{~N} / \mathrm{A}$ \\
\hline $\begin{array}{l}\text { E. colistrain: DH5 } \alpha \\
\text { fhuA2, } \Delta(\arg F-l a c Z) \cup 169, \text { phoA, glnV44, } \Phi 80 \text {, } \\
\Delta(\text { lacZ }) M 15, \text { gyrA96, recA1, relA1, endA1, thi-1, } \\
\text { hsdR17 }\end{array}$ & $\begin{array}{l}\text { Laboratory } \\
\text { strain }\end{array}$ & $\mathrm{N} / \mathrm{A}$ \\
\hline $\begin{array}{l}\text { E. colistrain: BW27749 } \Delta e n t F \\
\text { BW27749 strain with in-frame deletion of entF }\end{array}$ & This study & $\mathrm{N} / \mathrm{A}$ \\
\hline $\begin{array}{l}\text { E. coli strain: BL21 (DE3) } \\
\mathrm{F}^{-}, \text {omp T, gal, dcm, lon, hsdSB(rB- mB- ), } \lambda(\mathrm{DE} 3 \\
\text { [lacl, lacUV5-T7 gene } 1 \text {, ind1, sam7, nin5]) }\end{array}$ & 2 & $\mathrm{~N} / \mathrm{A}$ \\
\hline $\begin{array}{l}\text { E. colistrain: BL21 (DE3) ybdZ::aac(3)IV } \\
\text { BL21 (DE3) with ybdZ-disrupting apramycin } \\
\text { resistance cassette }\end{array}$ & 2 & $\mathrm{~N} / \mathrm{A}$ \\
\hline E. colistrain: MG1655 & ATCC & Cat \#700926 \\
\hline \multicolumn{3}{|l|}{ Primers } \\
\hline $\begin{array}{l}\text { pET28b-Ncol-5' } \\
\text { 5' - CATGGTATATCTCCTTCTTAAAGT }\end{array}$ & $\begin{array}{l}\text { Integrated DNA } \\
\text { Technologies }\end{array}$ & $\mathrm{N} / \mathrm{A}$ \\
\hline $\begin{array}{l}\text { pET28b-Hindlll-3' } \\
\text { 5' - AAGCTTGCGGCCGCACTCGAGCAC }\end{array}$ & $\begin{array}{l}\text { Integrated DNA } \\
\text { Technologies }\end{array}$ & $\mathrm{N} / \mathrm{A}$ \\
\hline $\begin{array}{l}\text { KTPdonDItAAF } \\
5^{\prime}- \\
\text { ACTITAAGAAGGAGATATACCATGATCACCG } \\
\text { ACCAGAGTTATC }\end{array}$ & $\begin{array}{l}\text { Integrated DNA } \\
\text { Technologies }\end{array}$ & $\mathrm{N} / \mathrm{A}$ \\
\hline $\begin{array}{l}\text { KTPdonDItATR } \\
\text { 5' - } \\
\text { GTGCTCGAGTGCGGCCGCAAGCTTAATGTA } \\
\text { GTCGACAATCGCC }\end{array}$ & $\begin{array}{l}\text { Integrated DNA } \\
\text { Technologies }\end{array}$ & $\mathrm{N} / \mathrm{A}$ \\
\hline $\begin{array}{l}\text { KTPdonDItAgF } \\
\text { 5' - GGCCTACTTGCCCTTGGATCC }\end{array}$ & $\begin{array}{l}\text { Integrated DNA } \\
\text { Technologies }\end{array}$ & $\mathrm{N} / \mathrm{A}$ \\
\hline $\begin{array}{l}\text { KTPdonDItAgR } \\
\text { 5'- AGTGCGATCAAGTITCCATGGTG }\end{array}$ & $\begin{array}{l}\text { Integrated DNA } \\
\text { Technologies }\end{array}$ & $\mathrm{N} / \mathrm{A}$ \\
\hline
\end{tabular}




\begin{tabular}{|c|c|c|}
\hline 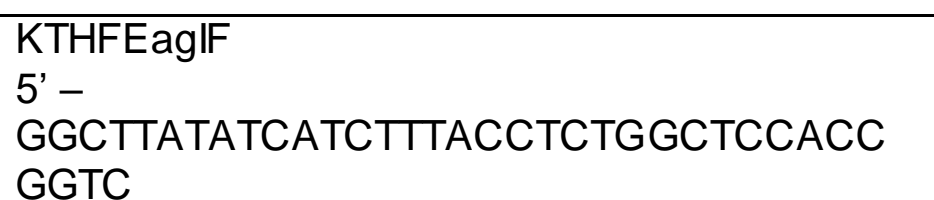 & $\begin{array}{l}\text { Integrated DNA } \\
\text { Technologies }\end{array}$ & $\mathrm{N} / \mathrm{A}$ \\
\hline $\begin{array}{l}\text { KTHFSaCIR } \\
\text { 5'- } \\
\text { CAATCGGCACACTGCTGCCGCGCACCTGTG } \\
\text { CGAGC }\end{array}$ & $\begin{array}{l}\text { Integrated DNA } \\
\text { Technologies }\end{array}$ & $\mathrm{N} / \mathrm{A}$ \\
\hline $\begin{array}{l}\text { KTEntF_site_2,3WT_Rev } \\
\text { 5'-GATAAACGGCCAGAAAAACTC }\end{array}$ & $\begin{array}{l}\text { Integrated DNA } \\
\text { Technologies }\end{array}$ & $\mathrm{N} / \mathrm{A}$ \\
\hline $\begin{array}{l}\text { KTEntF_site_2_Rev } \\
\text { 5' - } \\
\text { GATAAACGGCCAGAAAAACTCCCACACCGA } \\
\text { MNNATCAAAACTGCACGGCG }\end{array}$ & $\begin{array}{l}\text { Integrated DNA } \\
\text { Technologies }\end{array}$ & $\mathrm{N} / \mathrm{A}$ \\
\hline $\begin{array}{l}\text { KTEntF_site_3_Rev } \\
\text { 5' - } \\
\text { GATAAACGGCCAGAAAAACTCMNNCACCGA } \\
\text { GACATCAAAACTGCACGGCG }\end{array}$ & $\begin{array}{l}\text { Integrated DNA } \\
\text { Technologies }\end{array}$ & $\mathrm{N} / \mathrm{A}$ \\
\hline $\begin{array}{l}\text { VVEntF_site_2_3_Rev } \\
\text { 5'- } \\
\text { GATAAACGGCCAGAAAAACTCMNNCACCGA } \\
\text { MNNATCAAAACTGCACGGCG }\end{array}$ & $\begin{array}{l}\text { Integrated DNA } \\
\text { Technologies }\end{array}$ & $\mathrm{N} / \mathrm{A}$ \\
\hline $\begin{array}{l}\text { KTEntF_site_4_For } \\
\text { 5'-GAGTTTTCTGGCCGTTTATC }\end{array}$ & $\begin{array}{l}\text { Integrated DNA } \\
\text { Technologies }\end{array}$ & $\mathrm{N} / \mathrm{A}$ \\
\hline $\begin{array}{l}\text { KTEntF_site_4WT_Rev } \\
\text { 5'-GCCAGCATCGACGGCACAAAG }\end{array}$ & $\begin{array}{l}\text { Integrated DNA } \\
\text { Technologies }\end{array}$ & $\mathrm{N} / \mathrm{A}$ \\
\hline $\begin{array}{l}\text { KTEntF_site_4_Rev } \\
\text { 5'- } \\
\text { GCCAGCATCGACGGCACAAAMNNCGTGGTC } \\
\text { GTTACGCCATATTC }\end{array}$ & $\begin{array}{l}\text { Integrated DNA } \\
\text { Technologies }\end{array}$ & $\mathrm{N} / \mathrm{A}$ \\
\hline $\begin{array}{l}\text { KTEntF_site_5_6_For } \\
\text { 5'- TTTGTGCCGTCGATGCTGGC }\end{array}$ & $\begin{array}{l}\text { Integrated DNA } \\
\text { Technologies }\end{array}$ & $\mathrm{N} / \mathrm{A}$ \\
\hline $\begin{array}{l}\text { KTEntF_site_5,6WT_Rev } \\
\text { 5' - CAGCCGGTAAGGCCTCACC }\end{array}$ & $\begin{array}{l}\text { Integrated DNA } \\
\text { Technologies }\end{array}$ & $\mathrm{N} / \mathrm{A}$ \\
\hline $\begin{array}{l}\text { KTEntF_site_5_Rev } \\
\text { 5' - } \\
\text { CAGCCGGTAAGGCCTCACCACTACAMNNAA } \\
\text { CCTGTTICAACGTCGC }\end{array}$ & $\begin{array}{l}\text { Integrated DNA } \\
\text { Technologies }\end{array}$ & $\mathrm{N} / \mathrm{A}$ \\
\hline $\begin{array}{l}\text { KTEntF_site_6_Rev } \\
\text { 5'- } \\
\text { CAGCCGGTAAGGCCTCACCMNNACAGAAAA } \\
\text { CCTGTTTCAACGTCGC }\end{array}$ & $\begin{array}{l}\text { Integrated DNA } \\
\text { Technologies }\end{array}$ & $\mathrm{N} / \mathrm{A}$ \\
\hline $\begin{array}{l}\text { VVEntF_site_5_6_Rev } \\
\text { 5'- } \\
\text { CAGCCGGTAAGGCCTCACCMNNACAMNNAA } \\
\text { CCTGTTCAACGTCGC }\end{array}$ & $\begin{array}{l}\text { Integrated DNA } \\
\text { Technologies }\end{array}$ & $\mathrm{N} / \mathrm{A}$ \\
\hline
\end{tabular}




\begin{tabular}{|c|c|c|}
\hline $\begin{array}{l}\text { KTEntF_site_7_For } \\
\text { 5'-GGTGAGGCTTACCGGCTG }\end{array}$ & $\begin{array}{l}\text { Integrated DNA } \\
\text { Technologies }\end{array}$ & $\mathrm{N} / \mathrm{A}$ \\
\hline $\begin{array}{l}\text { KTEntF_site_7WT_Rev2 } \\
\text { 5' - CGCCGCTTCCGTCGGGCCATATAG }\end{array}$ & $\begin{array}{l}\text { Integrated DNA } \\
\text { Technologies }\end{array}$ & $\mathrm{N} / \mathrm{A}$ \\
\hline $\begin{array}{l}\text { KTEntF_site_7_Rev } \\
\text { 5' - } \\
\text { GCCGCTTCCGTCGGGCCATAMNNATTATGC } \\
\text { AACGGCGCGCCAG }\end{array}$ & $\begin{array}{l}\text { Integrated DNA } \\
\text { Technologies }\end{array}$ & $\mathrm{N} / \mathrm{A}$ \\
\hline $\begin{array}{l}\text { KTEntF_site_8,9WT_For } \\
\text { 5'- TATGGCCCGACGGAAGCGGCG }\end{array}$ & $\begin{array}{l}\text { Integrated DNA } \\
\text { Technologies }\end{array}$ & $\mathrm{N} / \mathrm{A}$ \\
\hline $\begin{array}{l}\text { KTEntF_site_8_For } \\
\text { 5' - } \\
\text { TATGGCCCGACGGAAGCGGCGNNKGATGTC } \\
\text { AGCTGGTATCCGGC }\end{array}$ & $\begin{array}{l}\text { Integrated DNA } \\
\text { Technologies }\end{array}$ & $\mathrm{N} / \mathrm{A}$ \\
\hline $\begin{array}{l}\text { KTEntF_site_9_For } \\
\text { 5'- } \\
\text { TATGGCCCGACGGAAGCGGCGGTANNKGTC } \\
\text { AGCTGGTATCCGGC }\end{array}$ & $\begin{array}{l}\text { Integrated DNA } \\
\text { Technologies }\end{array}$ & $\mathrm{N} / \mathrm{A}$ \\
\hline $\begin{array}{l}\text { VVaadAF } \\
\text { 5'- } \\
\text { GTGCCAACATAGTAAGCCAGTATACACTCCG } \\
\text { CTAGCGCCGTTGGAACACCAAGGAAAG }\end{array}$ & $\begin{array}{l}\text { Integrated DNA } \\
\text { Technologies }\end{array}$ & $\mathrm{N} / \mathrm{A}$ \\
\hline $\begin{array}{l}\text { VVaadAR } \\
\text { 5' - } \\
\text { GTCGCCTTGCGTATAATATTTGCCCATGGCT } \\
\text { TATTTGCCGACTACCTTGGTGATC }\end{array}$ & $\begin{array}{l}\text { Integrated DNA } \\
\text { Technologies }\end{array}$ & $\mathrm{N} / \mathrm{A}$ \\
\hline $\begin{array}{l}\text { rTBC0098 } \\
\text { 5'- } \\
\text { AATTACCTGTCACGATGACCGAGCTCGCACA } \\
\text { GGTGCGCGGCAG }\end{array}$ & $\begin{array}{l}\text { Integrated DNA } \\
\text { Technologies }\end{array}$ & $\mathrm{N} / \mathrm{A}$ \\
\hline $\begin{array}{l}\text { rTBC0099 } \\
\text { 5' - } \\
\text { GCGGTCATAAAGTCGACTTCCGGCCGACCG } \\
\text { GTGGAGCCAGAGGTAAAG }\end{array}$ & $\begin{array}{l}\text { Integrated DNA } \\
\text { Technologies }\end{array}$ & $\mathrm{N} / \mathrm{A}$ \\
\hline $\begin{array}{l}\text { rTBC0101 } \\
\text { 5' - GAAGTCGACTITATGACCGCCTC }\end{array}$ & $\begin{array}{l}\text { Integrated DNA } \\
\text { Technologies }\end{array}$ & $\mathrm{N} / \mathrm{A}$ \\
\hline $\begin{array}{l}\text { rTBC0102 } \\
\text { 5' - GGTCATCGTGACAGGTAATTACCC }\end{array}$ & $\begin{array}{l}\text { Integrated DNA } \\
\text { Technologies }\end{array}$ & $\mathrm{N} / \mathrm{A}$ \\
\hline $\begin{array}{l}\text { pACYC PIPE UP } \\
\text { 5'- ACACGGTGCCTGACTGCGTTAG }\end{array}$ & $\begin{array}{l}\text { Integrated DNA } \\
\text { Technologies }\end{array}$ & $\mathrm{N} / \mathrm{A}$ \\
\hline $\begin{array}{l}\text { pACYC PIPE DOWN } \\
\text { 5'- GGCGGCACCTCGCTAACG }\end{array}$ & $\begin{array}{l}\text { Integrated DNA } \\
\text { Technologies }\end{array}$ & $\mathrm{N} / \mathrm{A}$ \\
\hline $\begin{array}{l}\text { entF-pACYC PIPE UP } \\
\text { 5'- } \\
\text { CTAACGCAGTCAGGCACCGTGTATGAGCCA } \\
\text { GCATTTACCTTTGG }\end{array}$ & $\begin{array}{l}\text { Integrated DNA } \\
\text { Technologies }\end{array}$ & $\mathrm{N} / \mathrm{A}$ \\
\hline
\end{tabular}




\begin{tabular}{|c|c|c|}
\hline $\begin{array}{l}\text { entF-pACYC PIPE DOWN } \\
\text { 5' - } \\
\text { CGTTAGCGAGGTGCCGCCTTACCTGTTTAGC } \\
\text { GTTGCGC }\end{array}$ & $\begin{array}{l}\text { Integrated DNA } \\
\text { Technologies }\end{array}$ & $\mathrm{N} / \mathrm{A}$ \\
\hline $\begin{array}{l}\text { EntF-5'-Ncol28b } \\
\text { 5'-- } \\
\text { AGAAGGAGATATACCATGAGCCAGCATTTAC } \\
\text { CTITGGTCGCC }\end{array}$ & $\begin{array}{l}\text { Integrated DNA } \\
\text { Technologies }\end{array}$ & $\mathrm{N} / \mathrm{A}$ \\
\hline $\begin{array}{l}\text { EntF-3'-NoStop28b } \\
\text { 5' - } \\
\text { GAGTGCGGCCGCAAGCTTCCTGTTAGCGTT } \\
\text { GCGCGAATAAT }\end{array}$ & $\begin{array}{l}\text { Integrated DNA } \\
\text { Technologies }\end{array}$ & $\mathrm{N} / \mathrm{A}$ \\
\hline $\begin{array}{l}\text { KTPSSeq } 1 \\
\text { 5'- } \\
\text { TAGCGATGTTCCCAATTTAACAAGCCTTTGC }\end{array}$ & $\begin{array}{l}\text { Integrated DNA } \\
\text { Technologies }\end{array}$ & $\mathrm{N} / \mathrm{A}$ \\
\hline \multicolumn{3}{|l|}{ Plasmids } \\
\hline $\begin{array}{l}\text { pACYC duet- } y b d Z \\
\text { Coexpression of } y b d Z\end{array}$ & 2 & \\
\hline $\begin{array}{l}\text { pMAK705-entF } \\
\text { Temperature sensitive plasmid containing entF }\end{array}$ & This study & \\
\hline $\begin{array}{l}\text { pET28b } \\
\text { T7 expression vector }\end{array}$ & Novagen & Cat \#69865-3 \\
\hline $\begin{array}{l}\text { pET28bentF-ES-wildtype } \\
\text { Expression of EntF wild-type }\end{array}$ & This study & \\
\hline $\begin{array}{l}\text { pET28bdltA-wildtype } \\
\text { Expression of DltA wild-type }\end{array}$ & This study & \\
\hline $\begin{array}{l}\text { pET28bdltA-4-16 } \\
\text { Expression of DItA code variant 4-16 }\end{array}$ & This study & \\
\hline $\begin{array}{l}\text { pET28b-entF-ES-variant \# } \\
\text { Expression of EntF variants listed in Table } 1\end{array}$ & This study & \\
\hline $\begin{array}{l}\text { pCR-Bluntll-TOPO } \\
\text { For subcloning entF RS-encoding DNA }\end{array}$ & Invitrogen & Cat \#K280020 \\
\hline $\begin{array}{l}\text { pCR-Bluntll-TOPOentF-RS-F \#1-5 } \\
\text { Subcloning plasmids containing entF RS- } \\
\text { encoding DNA fragments } 1 \text { through } 5\end{array}$ & This study & \\
\hline $\begin{array}{l}\text { pACYC184 } \\
\text { Plasmid backbone used for all selections }\end{array}$ & ATCC & Cat \#37033 \\
\hline $\begin{array}{l}\text { pACYC184entF-ES } \\
\text { Contains wild-type entF }\end{array}$ & This study & \\
\hline $\begin{array}{l}\text { pACYC184entF-ES-RS sub } \\
\text { For cloning mutagenized entF libraries }\end{array}$ & This study & \\
\hline $\begin{array}{l}\text { pACYC184entF-ES-L1-4 } \\
\text { Plasmids containing libraries } 1-4\end{array}$ & This study & \\
\hline $\begin{array}{l}\text { pACYC184entF-ES-variant\# } \\
\text { Plasmids harvested from isolated ENT producer } \\
\text { strains listed in Table S2 }\end{array}$ & This study & \\
\hline
\end{tabular}




\section{References}

[1] Khlebnikov, A., Datsenko, K. A., Skaug, T., Wanner, B. L., and Keasling, J. D. (2001) Homogeneous expression of the P(BAD) promoter in Escherichia coli by constitutive expression of the low-affinity high-capacity AraE transporter. Microbiology 147, 3241-3247.

[2] Felnagle, E. A., Barkei, J. J., Park, H., Podevels, A. M., McMahon, M. D., Drott, D. W., and Thomas, M. G. (2010) MbtH-like proteins as integral components of bacterial nonribosomal peptide synthetases. Biochemistry 49, 8815-8817. 